\section{P429 GITELMAN SYNDROME - REPORT OF THE FIRST PEDIATRIC PATIENT FROM MACEDONIA}

${ }^{1}$ Aleksandra Janchevska*, 'Velibor Tasic, 'Olivera Jordanova, 'Zoran Gucev, Emma Ashton, ${ }^{2}$ Detlef Bockenhauer. 'University Children's hospital, Skopje, Macedonia, the former Yugoslav Republic of; ${ }^{2}$ Great Ormond Street Hospital for Children, London, UK

\subsection{6/archdischild-2019-epa.765}

Background and aims Gitelman syndrome (GS) is a rare renal channelopathy with an autosomal recessive type of inheritance, caused by homozygous or compound heterozygous mutations in SLC12A3 gene. The disorder causes metabolic abnormalities and may be overlapped with Barter type 3 syndrome.

Methods A 7 year-old boy presented with a history of several episodes of carpopedal spasms and muscle aches in the last few months. The boy has been a relatively healthy child previously. His auxological parameters were within normal range for his sex and age. Diagnostic assessment was achieved by characteristic symptoms, imaging technics, biochemical, hormonal and molecular analyses.

Results The biochemical analyses revealed hypokalemia (3.0$3.4 \mathrm{mmol} / \mathrm{l})$, hypomagnesemia $(0.64-0.66 \mathrm{mmol} / \mathrm{l})$, hypocalciuria $(<0.79 \mathrm{mg} / \mathrm{kg} / \mathrm{d})$ without metabolic alkalosis. The parathyroid and thyroid hormones were within reference range. The measured Q-T interval $(0.36 \mathrm{sec})$ in the electrocardiogram was also within normal range. No abnormalities were found in the audiogram and the electroencephalogram. The molecular analyses have shown that the patient was heterozygous for the SLC12A3 c.1805_1806del and c. $2660+1 \mathrm{G}>\mathrm{A}$ pathogenic variants and also heterozygous for the SLC12A3 c.2944 A>T (p. Ile982Phe) likely pathogenic variant. His mother was a heterozygous for the c.2944 A>T (p.Ile982Phe) mutation, but his father was heterozygous for the other 2 variants (SLC12A3 c. 1805 1806del and c. $2660+1 \mathrm{G}>\mathrm{A}$ ).

Conclusions Herein we present a first child from Macedonia with clinical manifestations and electrolyte imbalances typical for a GS. The results of the molecular analyses were consistent with the diagnosis of GS. The boy is on a high salt diet and oral potassium and magnesium supplements.

\section{P430 IDENTIFYING RARE DISEASE RESEARCH PRIORITIES THROUGH (RAINDROP) A RARE DISEASE RESEARCH PARTNERSHIP}

1,25uja Somanadhan*, 'Thilo Kroll, 'Emma Doris, 'Emma Nicholson, ${ }^{3}$ Sally Ann Lynch, ${ }^{3}$ Eileen Treacy, ${ }^{4}$ Aoife Brinkley, ${ }^{1}$ Sean Ennis, ${ }^{4}$ Atif Awan, Dr ${ }^{5}$ Avril Kennan, Vicky Mc Garth ${ }^{6},{ }^{7}$ Derick Mitchell. ${ }^{1}$ University College Dublin, Dublin, Ireland; ${ }^{2}$ Health Research Board, Dublin, Ireland; ${ }^{3} \mathrm{RCPI}$, Dublin, Ireland; ${ }^{4}$ Temple Street Hospital, Dublin, Ireland; ${ }^{5} \mathrm{MRCG}$ Dublin, Ireland; ${ }^{6}$ Rare Disease Ireland, Dublin, Ireland; ${ }^{7}$ IPPOSI, Dublin, Ireland

\subsection{6/archdischild-2019-epa.766}

Background To date, the rare disease research community is largely fragmented in Ireland without a coherent programmatic focus. All too often, priorities for research are decided by one group of stakeholders. The James Lind Alliance (JLA) model allows us to challenge this dynamic through the Rare Disease Research Partnership (RAinDRoP) Workshop which will enable clinicians, patients and carers to work together to identify and prioritise uncertainties that could be answered by research. The RAinDRoP is a truly collaborative effort in which everybody has a voice, and it refocuses the research on the patients rather than the disease.
Objectives To identify and prioritise topics and questions in the three research areas: (1) Route to Diagnosis (2) Living with rare diseases (3) Integrated care or Holistic care.

Design A modified nominal group technique.

Setting Republic of Ireland

Participants Anyone with experience of care for patients with rare diseases including patients, carers and healthcare professionals.

Results This workshop is scheduled to take place on the $04^{\text {th }}$ of April 2019. The result is not available at the time of submission.

Conclusions This study is the first national prioritisation exercise to identify patient and healthcare professional priorities for rare diseases research. The research priority partnership will identify a range of important gaps in the existing evidence to inform (1) Pathway to Diagnosis, focusing on facilitating early, accurate diagnosis and the communication/ informational challenges associated with a rare disease diagnosis (2) Supporting management and self-management of rare disease and (3) Integrated care pathways.

\section{P431 WHEN SICK ISN'T SEPTIC - METABOLIC CRISIS PRESENTING IN THE EARLY NEONATAL PERIOD}

Leah Halpenny*, Tracey Conlon, Aida Abedin, AA Monavari. National Centre for Inherited Metabolic Disorders, Children's University Hospital, Temple Street, Dublin, Ireland

\subsection{6/archdischild-2019-epa.767}

Introduction Metabolic crisis in the neonatal period is an infrequently encountered, but potentially devastating presentation of an inborn error of metabolism. Due to the rarity of these disorders, diagnosis may be delayed. Furthermore, presentation may closely mimic neonatal sepsis, a much more frequently encountered condition. We describe the case of a neonate who presented at day nine with non-specific symptoms and signs. Careful evaluation of biochemical abnormalities led to a timely diagnosis of a rare, potentially life threatening metabolic disorder - Argininosuccinic Aciduria.

Clinical Case A nine day old female infant presented with three days of poor feeding, lethargy and reduced urine output. Examination identified hypothermia (34.5C), tachypnoea, and a decreased level of consciousness. She was the first child of non-consanguineous Irish parents, with no family history of metabolic disorders. She was delivered at term by spontaneous vaginal delivery to non-consanguinous Irish parents, following an uncomplicated pregnancy. There were no septic risk factors or concerns at discharge on day 3 . Blood glucose and inflammatory markers were normal. She was fluid resuscitated and commenced on broad-spectrum antibiotics. Despite treatment, she deteriorated. Venous blood gas showed respiratory alkalosis $(\mathrm{pH}$ 7.458, pCO2 3.92, HCO3 20.8, BE -2.4, lactate 1.0) prompting further investigation. Serum ammonia was 562. Retrospective review of investigations identified an unrecordable urea $(<0.4 \mathrm{mmol} / \mathrm{L})$, and a diagnosis of urea cycle defect was considered. She was referred to the National Centre for Inherited Metabolic Disorders, and transferred to a tertiary intensive care unit for specialty input. Intravenous sodium benzoate, sodium phenylbutyrate and L-arginine were administered, alongside $10 \%$ dextrose. Further investigation revealed elevated glutamine of $2213 \mu \mathrm{mol} / \mathrm{L} \quad(0-960)$, citrulline $565 \mu \mathrm{mol} / \mathrm{L} \quad(0-63)$, and 